To appear in Perception

\title{
Orientation-Selective Adaptation during Motion-Induced Blindness
}

\author{
Leila Montaser-Kouhsari ${ }^{1}$, Farshad Moradi ${ }^{2}$, Amin Zandvakili ${ }^{1}$, \\ Hossein Esteky ${ }^{1,3}$
}

1) School of Cognitive sciences (SCS), Institute for Studies in Theoretical Physics and Mathematics (IPM), Tehran, Iran.

2) Computation and Neural Systems, California Institute of Technology, Pasadena, CA, USA

3) Research Laboratory for Brain and Cognitive Sciences, Shaheed Beheshti University, Tehran, Iran.

\begin{abstract}
When a global moving pattern is superimposed on high-contrast stationary or slowly moving stimuli, the latter occasionally disappear for periods of several seconds (Motion-Induced Blindness, MIB). In this study, an adaptation paradigm was used to determine if orientation-selective adaptation still occurs for the stimulus that is no longer visible. Two slowly-drifting, high-contrast Gabor patches were presented to observers. As soon as both patches disappeared, one was eliminated from the screen. After $2 \mathrm{~s}$, two low contrast Gabor patches were presented as tests at the same locations and observers were asked to report their orientations. The observers' performance was significantly higher when the orientation of low contrast test patch was orthogonal to the orientation of the high
\end{abstract}


contrast adapting patch $(\mathrm{p}<0.0001)$ for the location where the patch was present during MIB, even though it was perceptually invisible. The observers' performance was not significantly different at the adjacent control location where the stimulus was absent during the MIB. Although no stimulus was visible at either location, orientation selective adaptation was only preserved for the location at which the patch remained present. Since orientation information is processed in low-level visual areas like V1, we conclude that MIB originates in an area higher than V1.

Key Words: Motion induced blindness, Adaptation, Orientation and V1.

\section{Introduction}

Salient visual stimuli can be blocked from conscious perception under a number of special conditions such as spatial masking [Dehaene et al., 2001], spatial crowding [Toet \& Levi, 1992], binocular rivalry [Logothetis, 1998], attentional blink [Raymond et al., 1992], repetition blindness [Kanwisher, 1987], change blindness [Rensink et al., 1997], inattentional blindness [Mack \& Rock, 1998], and finally motion induced blindness (MIB) [Bonneh et al., 2001].

MIB is observed under normal viewing conditions. When a global moving pattern is superimposed on a high contrast stationary or slowly moving stimuli, the latter disappear and reappear alternatively for periods of several seconds. When two adjacent, collinear or parallel Gabor patches are presented, they disappear and reappear simultaneously but when two orthogonal Gabor patches are presented, their disappearances are independent [Bonneh et al., 2001]. 
The properties of MIB do not seem to reflect sensory suppression or adaptation, as opposed to the Troxler fading. First, highly supraliminal targets with high luminance contrast disappeared more often than low contrast targets. Therefore, MIB cannot be explained by a contrast-gain-control mechanism. Second, MIB does not always require prolonged presentation of the stimulus, and it sometimes occurs in a couple of seconds. Third, moving or dynamic targets may also disappear in MIB, producing the striking phenomenon of target dots that disappear in one quadrant and reappear in another after a few seconds. Such disappearance is unlikely to be explained by local adaptation or retinal stabilization effects. Finally, the effect does not depend on local masking, as targets continued to disappear even when surrounded by background-colored circular 'protection zones' that occluded the moving mask [Bonneh et al., 2001]. It has been argued that the disappearance of stimuli during MIB might reflect the higher-level attentional selection or disruption of attentional processing [Bonneh et al., 2001; Pettigrew \& Funk, 2001].

Visual adaptation has been repeatedly used for determining whether visual disappearance phenomena like binocular rivalry or spatial crowding occur in cortical areas higher than primary visual cortex (V1) [Blake \& Fox, 1974; He et al., 1996]. Preservation of orientation selective adaptation in either crowding or the suppression phase of binocular rivalry would suggest that these phenomena occur at a later stage than orientation processing in the visual stream (i.e. after V1).

We used an adapting paradigm to determine if an oriented stimulus that is perceptually invisible during MIB activates orientation-specific responses. If MIB 
originates after the level that orientation adaptation occurs, then it should not disrupt orientation-selective adaptation.

According to the adaptation paradigm, exposure to visual patterns of high-contrast Gabor patches creates adaptation of units tuned to the adapting orientation. Consequently, observers become temporarily insensitive to low-contrast test patterns of similar orientation [Blakemore \& Campbell, 1969]. In our experiment, observers viewed two adjacent parallel Gabor patches superimposed on a 'global moving pattern' (Figure 1). When the two patterns disappeared together, one of the Gabors was removed to serve as a control for the adaptation generated by the other. Nothing was visible at either location. If the disappearance lasted for $2 \mathrm{~s}$, the display was blanked and two low-contrast test stimuli were presented, either aligned with or orthogonal to the adapting orientations. The location that had no stimulus present during the two-second MIB interval served as a control for the location where the stimulus remained (but was invisible).

The two-second MIB interval is short compared to the time required for adaptation. However, we found that it is long enough for any adaptation that has built up over the previous exposure to dissipate. In particular, we observed in a control experiment (as described later) that, even following a long period of adaptation to visible Gabor patches (in the absence of global moving pattern), a two-second blank interval was sufficient for significant recovery from the orientation-specific adaptation. To be more specific then, our test examines whether the invisible Gabor maintains orientation-specific adaptation. 


\section{General Methods}

\section{Participants}

Five observers, 20-25 years old, with normal or corrected-to-normal vision, participated voluntarily in the experiments. All participants were trained psychophysical observers. They were, however, naive with respect to the purpose of the experiment.

\section{Apparatus}

The stimuli and the psychophysical experiment were programmed in Delphi 5 on a Pentium III $800 \mathrm{MHz}$ PC running Windows 98. Images were displayed on a RGB color monitor, $800 \mathrm{H}$ x $600 \mathrm{~V}$ pixel resolution at $60 \mathrm{~Hz}$ frame rate (795FT Plus, LG: Korea). The observers were placed in a dark room and viewed displays binocularly while their heads were fixed on a chin and forehead rest. The viewing distance was $40 \mathrm{~cm}$.

\section{Stimuli}

The MIB paradigm was designed using two adjacent parallel Gabor patches superimposed on a global moving pattern (See Figure 1).

The global moving pattern consisted of 180 black square dots, each $0.3^{\circ} \times 0.3^{\circ}$ in size, rotating in a random direction on an imaginary sphere, $15.4^{\circ}$ in diameter. The background of the moving pattern was gray with a mean luminance of $32 \mathrm{~cd} / \mathrm{m}^{2}$. A red crosshair fixation point was located on the bottom-right quadrant of the moving pattern. Adapting stimuli consisted of two parallel or co-axial Gabor patches superimposed on the top-left quarter of the sphere. The Gabor functions were 
composed of a Gaussian envelope with the standard deviation of 28 arcmin, with a cosine modulation of 28 arcmin wavelength (2.12 cycles per degree). Both Gabor patches were oriented either $45^{\circ}$ or $135^{\circ}$ within a "protection-zone" with diameter of $1.7^{\circ}$. The center-to-center distance between the adjacent patches was $3.2^{\circ}$ and the eccentricity of each Gabor patch from the fixation point was $10.2^{\circ}$. The peak contrast of the adapting stimuli was $100 \%$. To avoid local adaptation, the phase of each Gabor patch was slowly changed in a smooth motion over time ( 0.35 degree per second). Test stimuli consisted of two Gabor patches with the same properties of adapting stimuli but with peak contrast of $30 \%$. They could be either parallel or orthogonal. In a pilot experiment we found that this contrast for test stimuli was optimal for achieving a significant difference between the 'same-adapt Gabor patches' (Gabor patches with the same orientation as the adapting Gabor patches) and 'different-adapt Gabor patches' (Gabor patches with the different orientation from the adapting Gabor patches).

\section{Procedure}

Each trial began with the presentation of MIB display (see Figure 1). Gabor patches superimposed on the moving sphere were parallel/co-axial so they had a robust tendency to disappear and reappear simultaneously [Bonneh et al., 2001]. Observers were asked to press and hold down a key on the keyboard when the Gabor patches disappeared simultaneously and release it when either of them reappeared.

As soon as the observers pressed the key, the Gabor patch on the right side was eliminated from the screen and replaced by a homogeneous gray background while 
the left Gabor patch remained physically present. Subjects could not detect the elimination, as both patches were already perceptually invisible because of the MIB. The Gabor patch on the right side served as a control stimulus. Observers keep reporting the disappearance and reappearance of Gabor patches until they achieve a two-second MIB. Once the MIB lasted 2 s (i.e., the subject held down the key for $2 \mathrm{~s}$ ), the global moving pattern and the remaining adapting Gabor patch were eliminated and, after a delay interval of $300 \mathrm{~ms}$, the test stimuli were introduced.

The two test stimuli were presented for $500 \mathrm{~ms}$. Each test Gabor patch was oriented either same or orthogonal (with equal probability) with respect to the adapting orientation. Observers were asked to first report the orientation of the right test stimulus by pressing one of the two keys on the computer keyboard with their right hand, and then report the orientation of the left one with their left hand (two 2-AFC tasks). No feedback was given to the observers about their performance. The next trial began $2 \mathrm{~s}$ after a blank inter-trial interval.

Each subject performed 150 trials. The proportion correct of orientation discrimination was obtained for 'same-adapt Gabor patches' and 'different-adapt Gabor patches' separately. The mean time of adaptation period was calculated after pooling all data (i.e., the time elapsed for observers including the two-second MIB).

As the control condition, each participant performed another adaptation experiment using the calculated mean adaptation time obtained from the previous experiment. In this experiment adapting Gabor patches were presented on a gray background without any dots. This condition is a classic experiment of orientation selective 
adaptation in the absence of moving pattern. Two seconds before the end of adaptation phase, the right side Gabor patch was physically removed while the other patch remained. Observers again reported the orientations of test stimuli, just as in the previous experiment (see Figure 2).

Insert Figure 1 about here

Insert Figure 2 about here

\section{Results}

Proportion correct orientation discrimination in the test phase was compared between 'same-adapt' and 'different-adapt' conditions in the MIB and the control experiment. In the MIB experiment (after pooling all observers data), a two-way ANOVA revealed that the overall effect of adaptation (same-adapt vs. differentadapt, regardless of the presence of the stimulus during subjective MIB) was significant (Figure 3a, F $(1,1430)=99.60, \mathrm{p}<0.001)$. The effect of stimulus presence (physically invisible vs. perceptually invisible) was also significant ( $\mathrm{F}$ $(1,1430)=124.89, \mathrm{p}<0.001)$, and there was a significant interaction between adaptation and stimulus existence $(\mathrm{F}(1,1430)=65.27, \mathrm{p}<0.001)$. Post hoc analysis using Scheffé's test revealed significant difference between proportion corrects of 'same-adapt' and 'different-adapt' conditions of the perceptually invisible Gabor patches $(\mathrm{p}<0.0001$, Figure $3 \mathrm{a})$. In contrast, no significant difference was found 
between performances of 'same-adapt' and 'different-adapt' conditions of physically invisible Gabor patches $(\mathrm{p}=0.61)$.

The duration of adaptation period in the MIB condition was $8 \pm 5.3$ secs. Using a two-way ANOVA (pooled data), the effect of adaptation was found to be significant $(\mathrm{F}(1,670)=22.23, \mathrm{p}<0.001)$ in the control experiment. The effect of stimulus presence in the last $2 \mathrm{~s}$ was also significant $(\mathrm{F}(1,670)=19.87, \mathrm{p}<0.001)$. There was a significant interaction between adaptation and stimulus presence $(\mathrm{F}(1,670)=$ $11.58, \mathrm{p}<0.001)$. Post-hoc analysis showed that difference between performances of 'same-adapt' stimuli and 'different-adapt' stimuli was significant only for the stimulus that remained present during the last $2 \mathrm{~s}(\mathrm{p}<0.0001)$, whereas there was no significant difference for the stimulus that had been removed in the last $2 \mathrm{~s}(\mathrm{p}=$ 0.83 , Figure $3 b$ ). The effect on adaptation for the perceptually invisible Gabor in the MIB condition was very much like the effect of the visible patch in the control experiment. There was also no significant difference in adaptation to the left-side or right-side patch in the control experiment when both patches remained present during the adaptation period $(\mathrm{p}=0.15)$.

Insert Figure 3 about here 


\section{Discussion}

We demonstrated a robust maintenance of orientation-selective adaptation even when the adapting stimulus was perceptually invisible for $2 \mathrm{~s}$ due to MIB. In contrast, adaptation was weakened after 2 s during which the adapting stimulus was physically removed. This result showed that $2 \mathrm{~s}$ of delay time between adaptation and test phases were adequate for dissipating the adaptation even when the stimuli were presented for prolonged time during the adaptation phase. Such dissociation was also seen in the control experiment in which adapting stimuli were presented for a similar period.

Preservation of orientation selective adaptation for the perceptually-invisible Gabor patch during the MIB as well as for the remaining stimulus in the control experiment suggests that neurons responding to the stimulus orientation are still active during the disappearance phase of MIB.

The phase-shift of Gabor patches during the adaptation period prevented afterimage formation of adapting orientation in the delay period between adaptation and test phases and excludes the possibility of local adaptation in areas earlier than V1 (i.e. retina and LGN). V1 is the first site of orientation processing and it is a plausible candidate for being the first site of orientation selective adaptation of the oriented Gabor patches used in our study [Hubel \& Wiesel, 1997]. Since orientation adaptation occurs at or after V1, we conclude that the activity in V1 is not affected by MIB.

Orientation selective adaptation occurs even though observers do not have conscious access to the orientation information. This result is consistent with other 
findings suggesting that V1 activation alone does not necessarily determine conscious perception and V1 neurons can adapt even in the absence of awareness of the adapting stimuli [He et al., 1996; He \& MacLeod, 2001, Blake \& Fox, 1974]. However, disappearance from awareness might not necessarily be due to the suppression of the neuronal activity; it could be the result of decrease in synchronization. If MIB is the result of reduced synchronization in V1 then it might be possible to have adaptation when the disappearance happens. Even in this case, we have shown dissociation between the mere activity in V1 and the content of visual awareness. Further experiments are necessary to test if awareness may correspond to specific modes of cortical activity in V1 and other visual areas.

\section{Acknowledgments}

Authors wish to thank Patrick Cavanagh, Zoe Kourtzi, Ian Thornton, Shinsuke Shimojo, Reza Rajimehr and three anonymous reviewers for their helpful comments on the manuscript. This work was supported by School of Cognitive Sciences (SCS), Iranian Institute for Studies in Theoretical Physics and Mathematics (IPM), Tehran, Iran.

\section{References}

1) Blake R, Fox R (1974). Adaptation to invisible gratings and the site of binocular rivalry suppression. Nature, 249, 488-490

2) Blakemore C, Campbell F W (1969). Adaptation to spatial stimuli. Journal of Physiology. (Lond.) 200, 11P-13P

3) Bonneh Y S, Cooperman A, Sagi D (2001). Motion-induced blindness in normal observers. Nature, 411, 797-801 
4) Dehaene S, Naccache L, Cohen L, Bihan D L, Mangin J F, Poline J B, Riviere D (2001). Cerebral mechanisms of word masking and unconscious repetition priming. Nature Neuroscience 4, 752-758

5) He S, Cavanagh P, Intriligator J (1996). Attentional resolution and the locus of visual awareness. Nature, 383, 334-337

6) He S, MacLeod D I (2001). Orientation-selective adaptation and tilt after-effect from invisible patterns. Nature, 411, 473-476

7) Hubel D H, Wiesel T N (1977). Functional architecture of macaque monkey visual cortex. Proc. R. Soc. Lond. B 198, 1-59

8) Kanwisher N (1987). Repetition blindness: type recognition in token individuation. Cognition, 27, 117-143

9) Logothetis N K (1998). Single units and conscious vision. Philosophical Transactions of the Royal Society of London Series B - Biological Sciences, 353, 1801-1818

10) Mack A, Rock I (1998). Inattentional Blindness. Cambridge, MA: MIT Press

11) Pettigrew J D, Funk A P (2001). Opposing effects on perceptual rivalry caused by right vs. left TMS. Society for Neuroscience: Abstract

12) Raymond J E, Shapiro K L, Arnell K M (1992). Temporary suppression of visual processing in an RSVP task: an attentional blink? Journal of Experimental Psychology: Human Perception and Performance, 18, 849-860

13) Rensink R A, O'Regan J K, Clark J J (1997). To see or not to see: The need for attention to perceive changes in scenes. Psychological Science, 8, 368-373

14) Toet A, Levi D M (1992). The two-dimensional shape of spatial interaction zones in the parafovea. Vision Research, 32, 1349-1357 


\section{Figures}

Figure 1

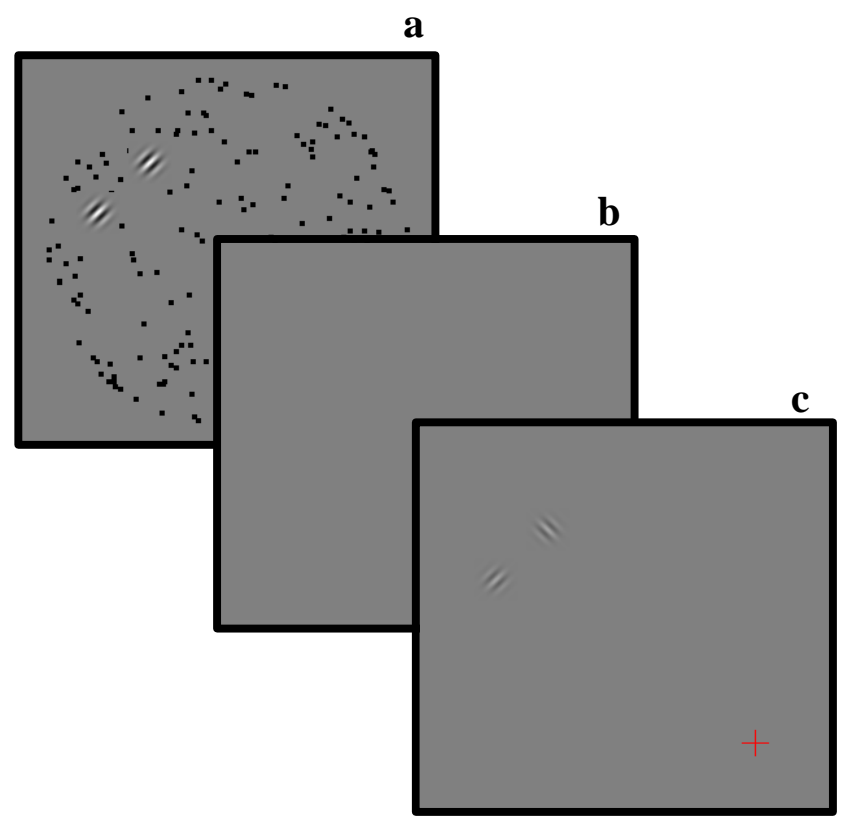


Figure 2

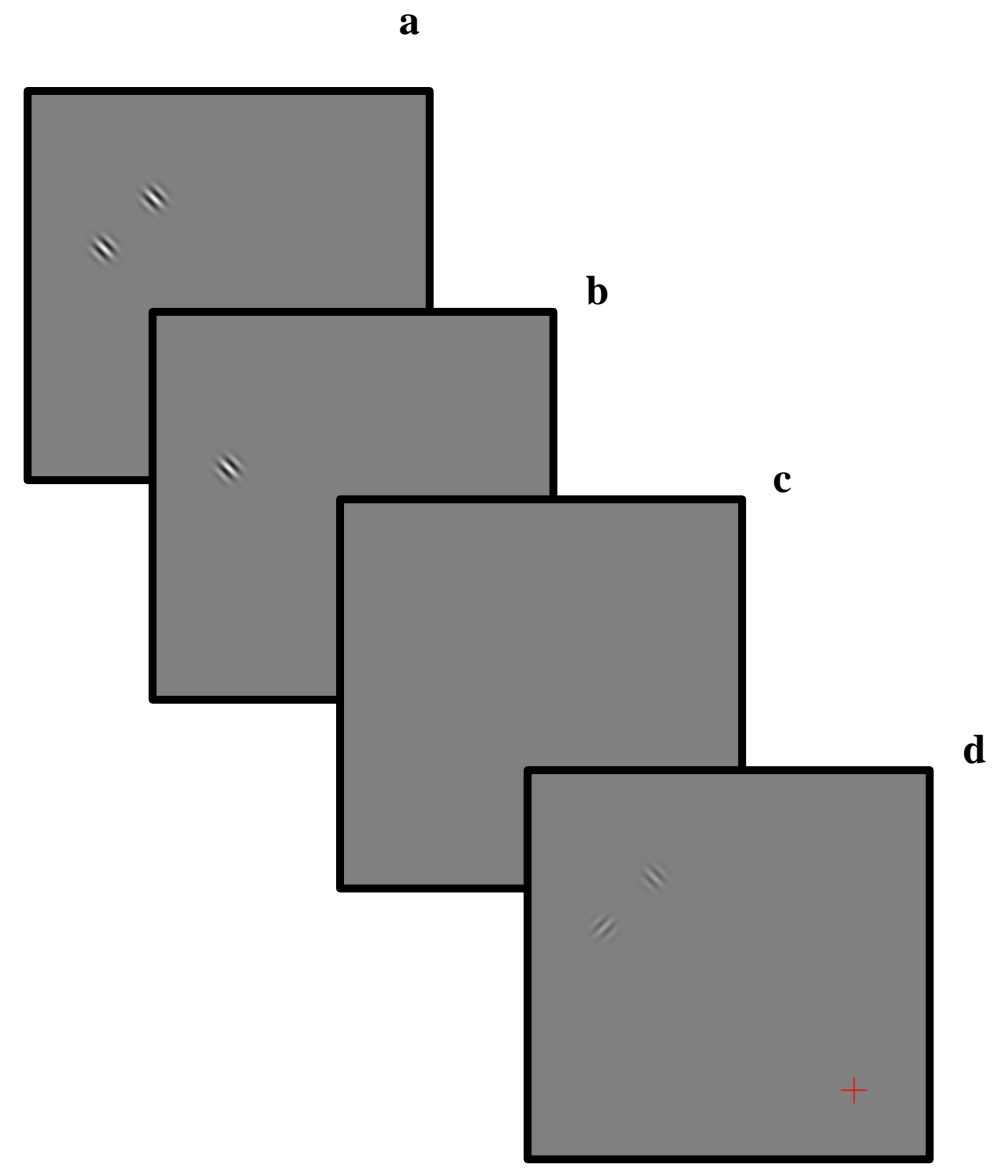


Figure 3

a)

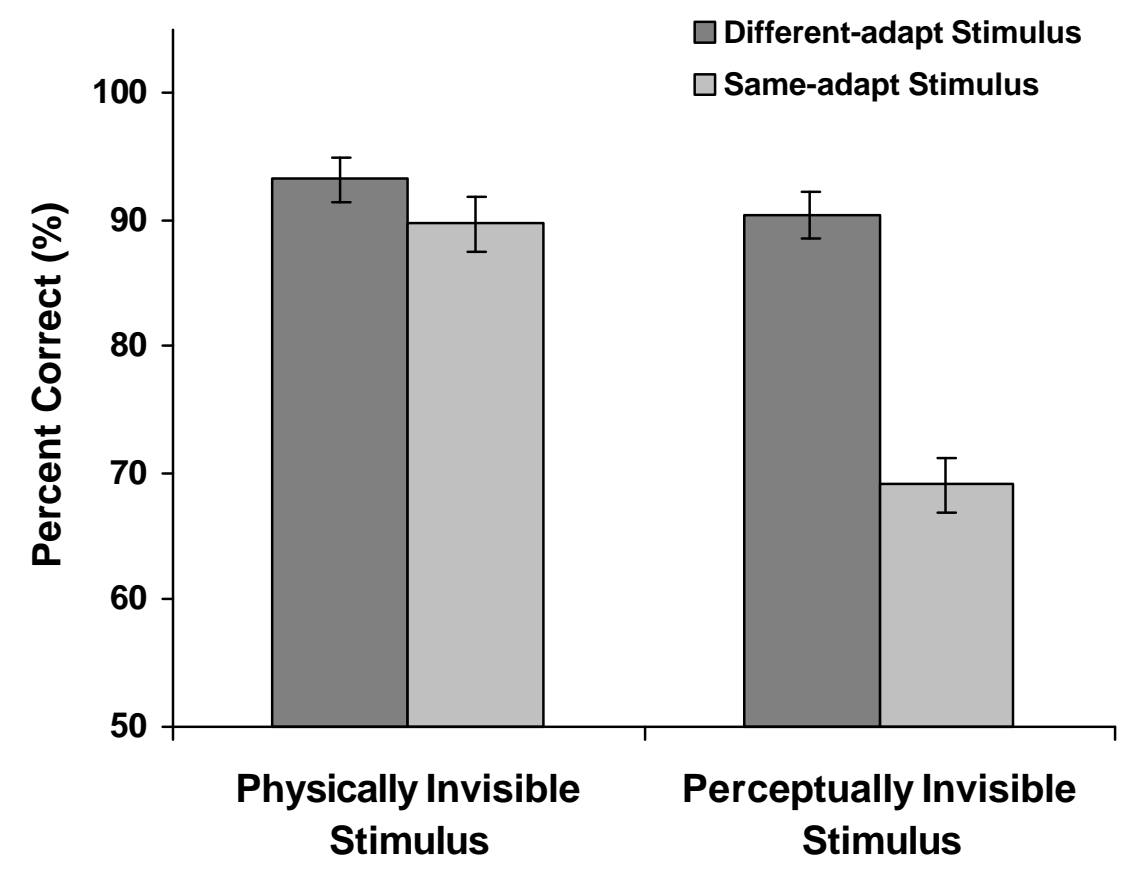

$\square$ Different-adapt Stimulus

$\square$ Same-adapt Stimulus

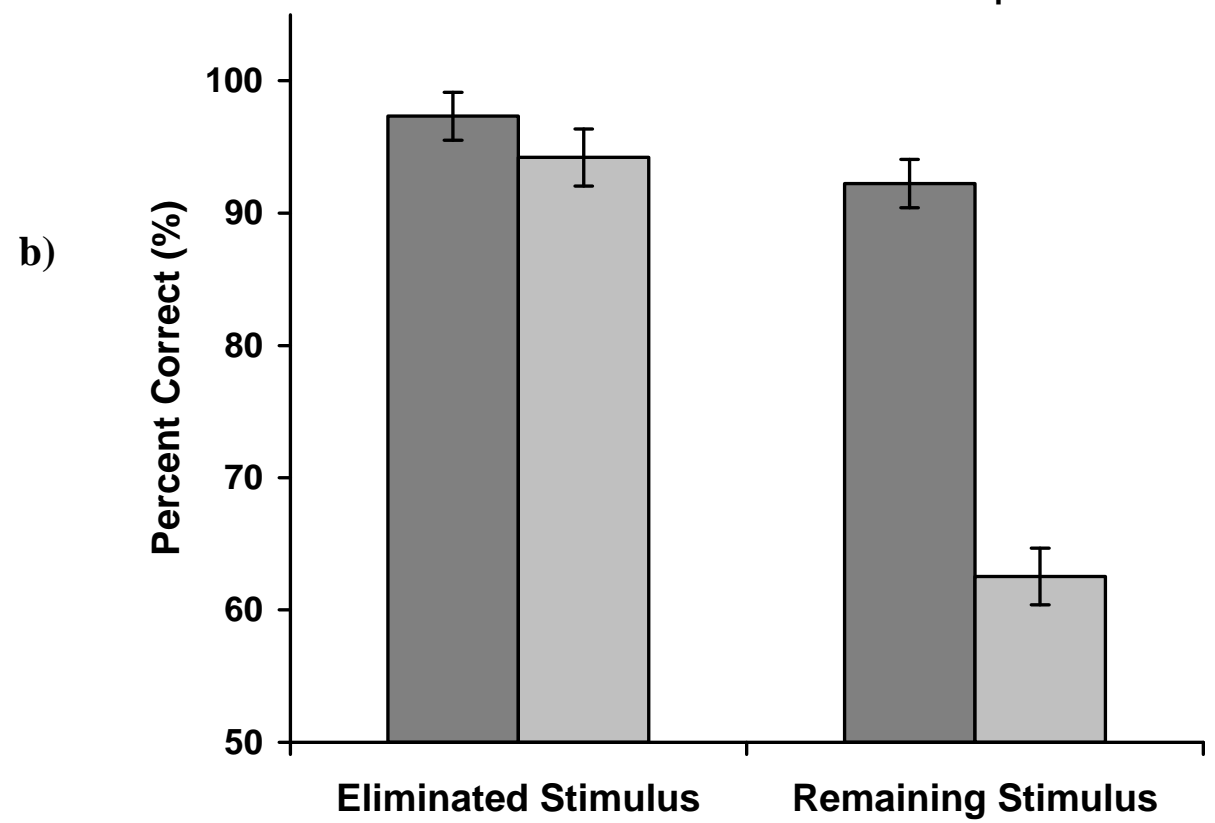




\section{Figure captions:}

Fig. 1 Schematic diagram demonstrating the temporal succession of visual stimuli in a typical trial of MIB experiment. a) The adaptation phase in the MIB condition consisted of two adjacent parallel/coaxial Gabor patches superimposed on the global moving pattern. b) Delay period between adaptation and test phases $(300 \mathrm{~ms})$. c) The test phase in which moving dots were removed and two test Gabor patches presented in the same locations of adapting stimuli for $500 \mathrm{~ms}$. The orientation of each test Gabor patch could be same or different with respect to the adapting orientation.

Fig. 2 a) The adaptation phase in the control experiment consisted of two adjacent parallel Gabor patches. b) Time delay of $2 \mathrm{~s}$ during which one of the Gabor patches was removed. c) Delay period between adaptation and test phases $(300 \mathrm{~ms})$. d) The test phase in which two tests Gabor patches presented in the same locations of adapting stimuli for $500 \mathrm{~ms}$. The orientation of each test Gabor patch could be same or different with respect to the adapting orientation.

Fig. 3 Performance of orientation discrimination for test stimuli in the a) MIB condition and b) control experiment. Perceptually Invisible adapting stimuli showed robust adaptation as well as the Remaining adapting stimuli but Physically Invisible adapting stimuli and Eliminated stimuli did not have any orientation-selective adaptation. 